\title{
ADOPTIVE TRANSFER OF IMMUNITY AGAINST RED MOUTH DISEASE IN RAINBOW TROUT (ONCORHYNCHUS MYKISS)
}

\author{
Takuya Yamaguchi, Uwe Fischer ${ }^{\S}$ \\ Federal Research Institute for Animal Health, Friedrich-Loeffler-Institut, \\ Südufer 10, 17393 Greifswald-Insel Riems, Germany
}

Enteric Red Mouth Disease (ERM) caused by Yersinia ruckeri is among the bacterial diseases in rainbow trout aquaculture that can be controlled by registered vaccines and part of these vaccines can be delivered by immersion which is the most convenient way of application inducing good antibody responses and ensuring decreased handling costs. However, the mechanisms by which fish are protected against Yersinia ruckeri are unclear. To shed light on this question we have bath vaccinated clonal rainbow trout donors by using a commercially available vaccine, and leukocytes have been isolated from vaccinated donors followed by adoptive cell transfer to naïve fish recipients of the same trout clone. Control recipients were injected with same numbers of leukocytes from non-vaccinated clonal donors. Transfer between genetically identical donors and recipients ensured that immune cells were not rejected by the recipients. After transfer, both groups of recipients were bath infected with a virulent field strain of Yersinia ruckeri. While rainbow trout that have received leukocytes from vaccinated donors showed no mortality, less than $40 \%$ of control recipients survived the challenge. Flow cytometry using leukocytes from recipient survivors revealed that the percentage of $\operatorname{IgM}^{+} \mathrm{B}$ cells was considerably higher in recipients that were injected with leukocytes from vaccinated donors when compared with control recipients, while no differences in the percentages of $\mathrm{CD} 8^{+}$cells among groups were recorded.

Being the first report on adoptive cellular transfer of immunity in a bacterial infection in fish these data suggest that immunity was rather transferred by $\operatorname{IgM}^{+} \mathrm{B}$ cells, but not by $\mathrm{CD} 8^{+}$cytotoxic $\mathrm{T}$ cells. . It can be further suggested that bath vaccination has caused an effective systemic immune response since protection could be transferred by non-mucosal leukocytes.

\section{KEYWORDS}

rainbow trout, Yersinia ruckeri, adoptive transfer, B cells, T cells

${ }^{\S}$ Corresponding author. Tel.: +49 3835171175 ; Fax: +49 3835171226.

E-mail address: uwe.fischer@fli.bund.de 